\title{
TAXONOMIC RELATIONSHIPS AMONG \\ DIATOM GENERA OF THE FAMILIES BIDDULPHIACEAE \\ AND EUPODISCACEAE FROM SEDIMENTS \\ OF THE GULF OF CALIFORNIA
}

\author{
RELACIONES TAXONOMICAS ENTRE \\ GENEROS DE DIATOMEAS DE LAS FAMILIAS BIDDULPHIACEAE \\ Y EUPODISCACEAE DE LOS SEDIMENTOS \\ DEL GOLFO DE CALIFORNIA \\ por/by \\ María del Carmen Chávez \\ $y /$ and \\ Timothy R. Baumgartner \\ CICESE \\ Centro de Investigación Científica y de Educación Superior de Ensenada \\ Èspiñoza No. 843 \\ Ensenada, Baja California, México
}

\begin{abstract}
A thorough review of the taxonomic relationships among the genera Biddulpbia, Odontella and Triceratium is presented. This includes a detailed description of the morphological features such as the ocellus, pseudocellus, costa, elevation, horn, spine and labiate process and the application of these structures in defining the position of species among similar genera. The study is directed towards the proper identification of diatoms from hemipelagic sediments of the Gulf of California, but is of general interest for the proper taxonomic placement of diatoms of the Biddulphiaceae and Eupodiscaceae families. A new combination, Odontella dubia, is proposed.
\end{abstract}

\section{RESUMEN}

Se presenta un examen concienzudo de las relaciones taxonómicas entre los géneros Biddulpbia, Odontella y Triceratium. Esto incluye una descripción detallada de rasgos morfológicos tales como ocellus, pseudocellus, costa, elevación, cuerno, espina y proceso labial y la aplicación de estas estructuras en la definición de la posición de especies entre géneros similares. El estudio está dirigido hacia la identificación apropiada de diatomeas de los sedimentos hemipelágicos del Golfo de California, pero es de interés general para la localización taxonómica apropiada de diatomeas de las familias Biddulphiaceae y Eupodiscaceae. Se propone una nueva combinación, Odontella dubia. 


\section{INTRODUCTION}

The need for this review became clear during a survey of established reference works, and the recent literature, to identify diagnostic characteristics of the various biddulphioid diatoms. As a necessary first step to the analysis of the recent phytoplankton community of the Gulf of California preserved within the sediments, the Department of Oceanography of CICESE is compiling a diagnostic catalog for use in the identification of the diatom species. This report represents a part of the effort applied to provide the correct taxonomic positions for species and genera encountered in that larger study.

Because the search for proper taxonomic positions of the biddulphioid diatoms has required considerably more effort compared to other groups of genera so far, it was felt that the taxonomic problems associated with these genera are worthy of this attention. The principal focus of this note is placed on the genera Biddulpbia, Odontella and Triceratium, although the discussion presents ramifications touching Cerataulus, Trigonium, Zygoceros and Hemiaulus. The morphological complexity and inherent difficulties in working with this group can be summarized by the statement of almost 20 years ago that, "The family Biddulphiaceae comprises a number of quite dissimilar generic groups, so much so, that it is almost impossible to give a clear circumscription of the characters" (Hendey, 1964, p. 99). More recently Hoban (1979, Table 2, p. 12-17) clearly presented the diversity of opinions which has existed from 1807 through 1975 regarding the taxonomic positions of these and related genera.

The purpose of this report is to identify the significant diagnostic features useful in separation of the genera (and in some cases, species) in which we are interested and provide a clarification of their taxonomic relationships based upon these characters. No new diagnostic characteristics are proposed here, rather we have attempted to summarize material from the existing literature. It should be noted that all characteristics discussed are visible by light microscopy. This is a practical requirement if the features are to achieve wide application for studies requiring rapid identification of species. However, a prior knowledge gained by study of published scanning electron microscope (SEM) photomicrographs is often necessary to use these characteristics with confidence under the light microscope.

The summary of diagnostic characters presented in this report has been applied only to those species in the sample material available for our study. Therefore the resulting classification is not comprehensive in the sense that species not present in the modern hemipelagic sediments of the Gulf of California are missing from the classification presented here. 


\section{MATERIAL USED}

The sample material examined during this study was taken from a onemeter-long box core recovered from the continental slope of the Guaymas Basin in the central Gulf of California. The core site is located in water depth of $670 \mathrm{~m}$ at $27^{\circ} 53.0^{\prime} \mathrm{N}, 111^{\circ} 35.5 \mathrm{~W}$, within the zone of the midwater oxygen minimum (Calvert, 1964). The inhibition of bioturbation by the poorly oxygenated water at the core site results in the preservation of seasonally formed laminae rich in well preserved diatom frustules (Calvert, 1966). Laminae counting and radiometric dating using Pb-210 from this core (Bruland,1974), indicate that all sample material examined here was deposited after the year 1825. Thus, all species preserved within the sediment obviously belong to the modern or existing phy toplankton community.

The individual samples examined in this study represent annual deposition of diatom frustules to the sea floor over the past 100 years. Individual laminae pair were sampled from the core after which the sediment was washed with deionized water. Hydrogen peroxide was then added to remove organic matter, heating the' samples for a period of 8 hours. After this treatment, the sample was sieved through $62 \mu \mathrm{m}, 24 \mu \mathrm{m}$ and $7 \mu \mathrm{m}$ mesh sizes. Permanent microscope slides were prepared from the size fraction $24 \mu \mathrm{m}\langle\mathrm{x}\langle 62 \mu \mathrm{m}$ and the $\rangle 62 \mu \mathrm{m}$ fraction. Diatoms were mounted on cover slips with Pleurax (Hanna, 1949). Examination of diatom mounts was carried out under Brightfield with a Zeiss Standard 18 microscope using oil immersion planachromat $(100 \mathrm{x})$ and planapochromat $(63 x)$ objectives. Drawings of the diatoms for figures 1-5 were done with camera lucida.

\section{BIDDULPHIOID CHARACTERISTICS}

Hendey (1964) provides an essential description of the main"biddulphioid" characteristics, although this must now be augmented by an awareness of recent work with the SEM advancing the understanding of the fine structure of diatom valves (e.g. Hoban, Fryxell, and Buck, 1980) and by the effort towards standardization of the terminology used for the morphological and structural characteristics of the diatom valves (Anonymous, 1975). According to Hendey (1964) the biddulphioid diatoms possess valves with a bipolar or polygonal outline whose angles or corners are modified "either to form a 'process', 'horn', or 'spine' or to be slightly raised above the general valve level"; "spines, one, two or many", are usually observed on the valve surface; the height of the girdle is normally equal to or greater than the length of the transapical axis (or width of the valve). This summary of characteristics must be revised to agree with the proposal for standardized definition and terminology given by Anonymous (1975) and to clarify the descriptions of the genera. Hendey's (1964) use of the terms "process", "horn" and "spine", as well as his reference to the valve angles "slightly raised above the general valve level" are now somewhat confusing and outdated. 
Anonymous (1975) defines "elevation" as a "raised portion of the valve wall, not projecting laterally beyond the valve margin, that may bear on it some special structure, but otherwise has much the same structure as the valve". Examples of elevations on a bipolar valve are seen in Biddulpbia biddulpbiana, illustrated in figure 1; elevations on the surface of the polygonal valve of Odontella dubia are indicated in figure 3. Unlike a process or spine, a "horn" is considered a special case of a modified elevation "that is long and narrow". The two outer projections along the valve margin of Odontella mobiliensis (Figures 4,12 , and 13) are good examples of horns. "Spines" (Figures 5 and 8 ) are considered by Anonymous (1975) as one of several structures included under "processes". A process is described as a projection from the valve wall which itself possesses "homogeneously silicified walls" and a "spine" is further defined as a "closed or solid structure projecting out from the wall". The term "process" also includes a structure described as "labiate process" which is an external tube or "opening through the valve wall with an internal flattened tube or longitudinal slit often surrounded by two lips" on the inside of the valve (Anonymous, 1975). When Hendey (1964) wrote his description of biddulphioid characteristics, the labiate process structure had not yet been clearly recognized and described. G. Fryxell and M. Hoban (personal communication; Hoban, 1979) also note that the apparent long spines (Figures 4 and 5 ) in the genus Odontella are actually the external tubes of labiate processes compared to similar processes in the genus Hemiaulus which are true spines and not labiate processes, and are thus separate structures.

The SEM study by Ross and Sims (1971) of fine structure of the valve provides the soundest foundation for defining the genera within the group of biddulphioid diatoms. Their work has indeed demonstrated that a number of genera, thus far considered to be members of the family Biddulphiaceae, are more naturally associated with the family Eupodiscaceae. They point out that this confusion in systematics has occurred in part as the result of the great importance which many early workers gave to the shape of the valve outline and general morphology of the valve; in particular, whether the valve is bipolar or multiangular (polygonal). Hustedt (1930) is cited by Ross and Sims (1971) as placing particular emphasis on the difference between bipolar and multiangular symmetry. Their study disputed this strict reliance of earlier authors on the symmetry of valve outlines to place generic limits on the biddulphioid diatoms. They single out Hendey (1964) as the only modern authority who has proposed a generic classification of the family Biddulphiaceae which is not strongly based on valve symmetry. Thus Hendey placed many polygonal species within the genus Biddulphia, which had been restricted to bipolar species by Hustedt (1930), although he still limited Triceratium to species with valves of three or more angles.

\section{TAXONOMIC RELATIONSHIP OF BIDDULPHIA, ODONTELLA, AND TRI- CERATIUM}

Examination of ten species by Ross and Sims (1971) previously considered as belonging to the genera Biddulpbia, Triceratium and Cerataulus, all within the 
family Biddulphiaceae, established the following taxonomic groups of either one or two genera:

1) Valves porose; markings on valves grading into those on processes or angles.

2) Valves alveolate; markings on valve grading into those on processes or angles.

3) Valves porose; processes or angles with finely perforate plate surrounded by a solid rim.

4) Valves alveolate; processes or angles with finely perforate platc surrounded by a solid rim.

The first two groups possess processes which are described by Anonymous (1975) as pseudocellate, while the processes of the third and fourth groups are described as ocellate. An ocellus (Figurcs 2, 3, 4, 5, 6, 7, 10) occurs at the end of a horn or elevation and is a "plate of silica, normally a thickened, structureless rim, pierced by closely packed holes" (Anonymous, 1975). A pseudocellus has the superficial appearance of an ocellus but results from the reduction in size of areolae along the hom or elevation away from the main part of the valve. This rcduction in areolae size is indicated on the left elevation of the drawing of $B$. biddulphiana (Figure 1 ) and can be seen on the angles of the valve of $B$. alternans illustrated in figure 15. Ross and Sims (1971) conclude that the first group (poroid areolae, pseudocellate) corresponds to the single genus Biddulpbia; the second group (alveolate areolae, pseudocellate), is limited to the genus Trigonium; the third group (poroid areolae; ocellate) corresponds to the single genus Odontella; the fourth group (alveolate areolae; ocellate) to the two genera Zygoceros and Cerataulus. They further suggested that the classification into higher groups (families) be based upon the ocellate versus pseudocellate structure, thus separating their genera of Odontella (along with Zygoceros and Cerataulus) in to the Eupodiscaceae family and Biddulpbia (along with Trigonium) into the Biddulphiaceae family. The original Triceratium species examined were then included within all of these genera.

Although Simonsen (1974) agreed with Ross and Sims (1971) that the use of valve symmetry had been "overrated" as a diagnostic taxonomic character, he chose to maintain the distinction between bipolar and tri-- or polygonal genera. He did however, accept the Ross and Sims (1971) conclusion that those genera which were earlier considered to belong to the family Biddulphiaceae, but which possess ocelli, should be placed in the family Eupodiscaceae. Thus many of the "biddulphioid" species which are encountered within our sample material from the Gulf of California, and which have been traditionally placed within the genus Biddulphia by Hendey (1964) and by Hustedt (1930) should be moved to the genus Odontella because they possess ocelly. These species are listed in Table 1 along with their synonyms, references for identification and location within the figures of this study if illustrated here. In this definition of generic limits we are thus following the fundamental elements of Ross and Sims (1971) observations 
TAXONOMIC RELATIONSHIPS DIATOM GENERA

which were adhered to by Simonsen (1974) for these species, and confirmed by Hoban (1979).

All of the species epithets listed in Table 1 under the genus Odontella are known by us to appear in the previous literature except $O$. dubia. We have decided to transfer it from Biddulpbia dubia after the comparison of diagnostic features in Triceratium dubium (this species earliest name), Triceratium favus (type of the genus) and Biddulpbia dubia as illustrated by Allen and Cupp (1935), which closely resembles the characteristics of our specimens.

Hoban (1979) observed in T. dubium a poroid valve structure and pseudoloculations similar in structure to those of $T$. favus, but less regular and extending completely over the valve. A pseudoloculus (Figure 2) is described by Anonymous (1975) as a "chamber formed on the outer side of the basal siliceous layer by expansion of the distal parts of anastomosing or reticulate costae". Hoban (1979) also observed that the elevations of $T$. dubium are ocellate. Cupp (1943) sketched her $B$. dubia with wavy and irregular reticulations of unequal size on the valve surface, bipolar and ocellate valves, with "the sides extended to give a nearly square cross sections", and the center part of the valve convex. Hendey (1964) states that the valve surface of $T$. favus is covered with a regular hexagonal loculation or reticulation arranged in straight lines parallel to the valve margins. Ross and Sims (1971) also report hexagonal and regularly arranged chambers in the outer side of the valve of $T$. favus.

Specimens of our O. dubia from the Gulf of California show the following principal characteristics: 1) poroid areolae, 2) wavy and irregular reticulations (pseudoloculae) of unequal size on the valve surface, 3) opposing ocellate elevations, 4) valve may be polygonal in outline due to extension of the two angles with opposing non-ocellate elevations, each which support a horn-like projection (Figures 2, 3,9 and 11). All of these characteristics are found in Allen and Cupp's (1935) B. dubia, and 1), 2) and 3) are shared with Hoban's (1979) T. dubium. However, our specimens do not have the regular hexagonal loculation of $T$. favus (Hendey, 1964) and they possess the horn-like projections from the two nonocellate elevations; these projections are not mentioned by Hoban from his description of $T$. dubium nor do they appear to be present from his figures. Although we have not examined our specimens of Odontella dubia under the SEM, we believe that these prominent projections are labiate processes. It should also be noted that Cupp's (1943) description of B. dubia does not agree with that of Allen and Cupp (1935). She seems to have mislocated the position of the two horn-like projections on her figure 114 where they appear as heavy spines each projecting from the base of an ocellate elevation. The foregoing observations indicate that the specimens are definitely what Allen and Cupp (1935) described as $B$. dubia. However, we believe that the ocellate elevations require that it be removed from the genus Biddulpbia and placed within Odontella.

Within the sample material from the Gulf of California, there have been 
found two species which are pseudocellate and should therefore be placed within the genus Biddulpbia. These are B. biddulpbiana and B. alternans; they are listed (Table 2) with their synonyms plus references for identification and are illustrated in the figures 1,14 , and 15. Note that we do not agree with Simonsen (1974) to maintain genera based upon valve symmetry. B. alternans is tripolar (Figure 15) and is equivalent to the Trigonium altermans of Simonsen (1974) and Triceratium alternans of Hustedt (1930). However, it is pseudoccllate and possesses characteristics which parallel those of the genus Biddulpbia described by Hendey (1964). It has well developed costae and is coarsely areolate; moreover, its arrangement of areolae may be similar to that of $B$. biddulphiana. Hoban (1979) also notes the strong similarity between $B$. alternans and $B$. biddulpbiana (which is the type species of the genus Biddulpbia). Hendey (1964) described the principal characteristics of $B$. alternans as "the valve being divided into three or more sections by strong costae" which are "usually transverse"; and the valve surface between the costae is "coarsely areolate, reticulate, areolate rounded, arranged in longitudinal or transverse rows towards the ends, but concentric or irregular in the center". Biddulphia biddulphiana is given the epithet $B$. pulchella by Hustedt (1930), Simonsen (1974), Hendey (1964). However, Hoban (1979) argues that $B$. biddulphiana is the correct name and we have elected to follow his criteria for the species name.

In addition to the use of ocelli and pseudocelli for separation of the Biddulpbia and Odontella genera (and thus their families), the structure of labiate processès may also be useful, although Hoban (1979) is careful to note that they are less reliable at the familiar level.

Hoban (personal communication) has noted that the labiate processes of "most Biddulpbia" have no external tubes while "most Odontella" do. Hoban (1979) observed that $B$. biddulpbiana usually has no labiate processes but sometimes between two and six are found in the central region of the valve; $B$. alternans possesses three midway between the elevation. In Odontella the labiate processes are the apparent long spines (Figures 4, 12 and 13; external tubes) which may be close to the center, or near the margin of the valve; both their number and placement can be quite variable within a single species.

\section{RELACIONES TAXONOMICAS ENTRE \\ GENEROS DE DIATOMEAS DE LAS FAMILIAS BIDDULPHIACEAE Y EUPODISCACEAE DE LOS SEDIMENTOS DEL GOLFO DE GALIFORNIA}

\section{INTRODUCCION}

La necesidad para este examen se hizo evidente durante una revisión de trabajos de referencia ya establecidos, y de la literatura reciente para identificar características de diagnóstico de las diferentes diatomeas biddulphioides. Como un 
primer paso necesario para el análisis de la comunidad fitoplanctónica reciente del Golfo de California preservada dentro de los sedimentos, el Departamento de Oceanografía de C I C E S E está recopilando un catálogo de diagnóstico para uso de la identificación de especies de diatomeas. Este reporte representa una parte del esfuerzo aplicado para determinar las posiciones taxonómicas correctas para especies y géneros encon trados en ese estudio mayor.

Debido a que la búsqueda de posiciones taxonómicas apropiadas de las diatomeas biddulphioides ha requerido de un esfuerzo considerablemente mayor, hasta ahora, comparado con otros grupos de géneros, se consideró que los problemas taxonómicos asociados con estos géneros son merecedores de esta atención. El foco principal de esta nota está colocado en los géneros Biddulpbia, Odontella y Triceratium, aunque la discusión presenta ramificaciones que tocan a Cerataulus Trigonium, Zygoceros y Hemiaulus. La complejidad morfológica y las dificultades inherentes al trabajar con este grupo, se pueden resumir en el postulado de hace casi 20 años de que, "La familia Biddulphiaceae comprende un número de grupos genéricos bastantes disímiles, tanto, que es casi imposible delimitar claramente sus caracteres" (Hendey, 1964, p. 99). Más recientemente Hoban (1979, Tabla 2, p. 12-17) presentó claramente la diversidad de opiniones que ha existido desde 1807 a 1975 en relación a las posiciones taxonómicas de estos géneros y otros relacionados.

El propósito de este reporte es identificar los rasgos significativos de diagnóstico útiles para la separación de los géneros (y en algunos casos, especies) en los que estamos interesados, y proveer una aclaración de sus relaciones taxonómicas basadas en estos caracteres. No se proponen aquí nuevas características de diagnóstico, más bien hemos intentado resumir el material de la literatura existente. Deberá hacerse notar que todas las características discutidas son visibles con el microscopio de luz.

Este es un requisito práctico si se desea que los rasgos alcancen una aplicación amplia para estudios que requieran una identificación rápida de especies. Sin embargo, frecuentemente es necesario un conocimiento previo alcanzado por el estudio de fotomicrografías de Microscopio de Barrido Electrónico ("SEM") publicadas, para utilizar estas características con confianza bajo el microscopio de luz.

El resumen de características de diagnóstico presentado en este reporte se ha aplicado sólo a aquellas especies encontradas en el material de muestreo disponible para este estudio. Por lo que la clasificación resultante no es comprensiva en el sentido de que las especies no presentes en los sedimentos hemipelágicos modernos del Golfo de California están ausentes en la clasificación que aquí se presenta. 


\section{MATERIAL UTILIZADO}

El material de muestreo examinado durante este estudio se tomó de un núcleo de caja de un metro de longitud recuperado de la plataforma continental de la Cuenca de Guaymas en la parte central del Golfo de California. El sitio nucleado se localiza a una profundidad marina de $670 \mathrm{~m}$, a $27^{\circ} 53.0^{\prime} \mathrm{N}, 111^{\circ} 35.5^{\prime} \mathrm{W}$, dentro de la zona del mínimo de oxígeno de media agua (Calvert, 1964). La inhibición de la bioturbidez, ocasionada por el agua pobremente oxigenada en el sitio nucleado, da como resultado la preservación de laminillas de origen estacional ricas en frústulas de diatomeas muy bien preservadas (Calvert, 1966). El conteo de laminillas y el datado radiométrico utilizando $\mathrm{Pb}-210$, de este núcleo (Bruland, 1974), indican que todo el material que aquí se cxaminó fuc depositado después del año 1825. Por lo que todas las especies preservadas dentro del sedimeno, obviamente pertenecen a la comunidad fitoplanctónica moderna o existente.

Las muestras individuales examinadas en este estudio representan la depositación anual hacia el fondo del océano de frústulas de diatomeas durante los últimos 100 años. Se muestrearon pares individuales de laminillas del núcleo, después de lo cual se lavó el sedimento con agua deionizada. Se le añadió entonces peróxido de hidrógeno para deshacer la materia orgánica, calentando las muestras por un período de 8 horas. Después de este tratamiento, se coló la muestra a través de mallas de $67 u \mathrm{~m}, 24 u \mathrm{~m}$ y $7 u \mathrm{~m}$. Se prepararon placas permanentes para microscopio a partir de la fracción $24 \mu \mathrm{m}\langle\mathrm{x}\langle 62 \mu \mathrm{m}$ y de la fracción $>62 \mu \mathrm{m}$. Se montaron las diatomeas con Pleurax (Hanna, 1949) sobre cubreobjetos. El examen de montajes de diatomeas se llevó a cabo bajo luz brillante con un microscopio Estandar Zeiss 18, utilizando los objetivos de aceite de inmersión planachromat $(100 x)$ y planapochromat $(63 \mathrm{x})$. Los dibujos de diatomeas para las figuras $1-5$ se hicieron con cámara lúcida.

\section{CARACTERISTICAS BIDDULPHIOIDES}

Hendey (1964) provee una descripción esencial de las principales características "biddulphioides", aunque ésto deba ahora complementarse con el reconocimiento de trabajos recientes con el Microscopio de Barrido Electrónico, que promueven el conocimiento de la estructura fina de las valvas de diatomeas ( e. g. Hoban, Fryxell, y Buck, 1980), y el esfuerzo hacia la normalización de la terminología utilizada para las características morfológicas y estructurales de las valvas de diatomeas (Anonymous, 1975). De acuerdo a Hendey (1964) las diatomeas biddulphioides poseen valvas con contorno bipolar o poligonal, cuyos ángulos o esquinas están modificadas "ya sea para formar un 'proceso', 'cuerno', o 'espina' o para ser ligeramente elevados sobre el nivel general de la valva", "las espinas, una, dos o muchas", se observan comúnmente en la superficie de la valva; la altura del cinturón es normalmente igual a o mayor que la longitud del eje transapical (o ancho de la valva). Este resumen de características deberá revisarse para que 
concuerde con la proposición para la definición y terminología normalizadas dadas por Anonymous (1975) y para aclarar las descripciones de los géneros. El uso de Hendey (1964) de los términos "proceso", "cuerno" y "espina", tanto como su referencia a los ángulos de la valva "ligeramente elevados por encima del nivel general de la valva" son ahora un tanto confusos y fuera de época.

Anonymous (1975) define una "elevación" como una "porción elevada de la pared de la valva, que no se proyecta lateralmente más allá del margen de la valva, y que puede presentar alguna estructura especial, pero que por otra parte tiene bastante similitud con la estructura de la valva". Algunos ejemplos de elevaciones en una valva bipolar se ven en Biddulpbia biddulpbiana, ilustrada en la figura 1; las elevaciones en la superficie de la valva poligonal de Odontella dubia se indican en la figura 3. A diferencia de un proceso o espina, un "cuemo" está considerado como un caso especial de una elevación modificada "que es larga y angosta". Las dos proyecciones exteriores a lo largo del margen de la valva de Odontella mobiliensis (figura 4, 12 y 13) son buenos ejemplos de cuernos. Las "espinas" (figuras 5 y 8) están consideradas por Anonymous (1975) como una de varias estructuras incluidas bajo "procesos". Un proceso se describe como una proyección de la pared de la valva que posee en sí "paredes homogéneamente silicificadas" y una "espina" se define posteriormente como una "estructura sólida o cerrada que se proyecta hacia afuera de la pared". El término "proceso" incluye además una estructura descrita como "proceso labial", que es un tubo externo o "apertura a través de la pared de la valva con un tubo interno aplanado o ranura longitudinal comúnmente rodeada por dos labios" en el interior de la valva (Anonymous, 1975). Cuando Hendey (1964) escribió su descripción de características biddulphioides, la estructura proceso labial no había sido aún claramente reconocida y descrita. G. Fryxell y M. Hoban (comunicación personal, Hoban, 1979) hacen también notar que las aparentes espinas largas (figuras 4 y 5 ) en el género Odontella son realmente los tubos externos de procesos labiales, comparados a procesos similares en el género Hemiaulus, que son verdaderas espinas y no procesos labiales, y son por lo tanto estructuras diferentes.

El estudio de Ross y Sims (1971) con Microscopio de Barrido Electrónico de la estructura fina de la valva, provee el fundamento más sólido para definir los géneros dentro del grupo de diatomeas biddulphioides. Su trabajo ha demostrado realmente que un número de géneros, hasta ahora considerados como miembros de la familia Biddulphiaceae, están asociados más naturalmente con la familia Eupodiscaceae. Señalan que esta confusión en sistemática ha ocurrido en parte como resultado de la gran importancia que muchos estudiosos de antaño dieron a la forma del contorno de la valva y a la morfología general de la valva; en particular, a si la valva es bipolar o multiangular (poligonal). Hustedt (1930) es citado por Ross y Sims (1971) como alguien que puso particular énfasis en la diferencia entre la simetría bipolar y la multiangular. El estudio de Ross y Sims disputó esta estricta dependencia de los primeros autores en la simetría de los contornos de la valva para colocar límites genéricos en las diatomeas biddulphioides. Ross y 
Sims (1971) eligen a Hendey (1964) como la única autoridad moderna que ha propuesto una clasificación genérica de la familia Biddulphiaceae, que no está fuertemente basada en la simetría de la valva. Por lo que Hendey coloca a muchas especies poligonales dentro del género Biddulpbia, que había sido restringido a especies bipolares por Hustedt (1930), a pesar de que limitó aún a Triceratium a especies con valvas de tres o más ángulos.

\section{RELACIONES TAXONOMICAS DE Biddulpbia, Odontella $y$ Triceratium.}

El examen llevado a cabo por Ross y Sims (1971) de diez especies anteriormente consideradas como pertenecientes a los géneros Biddulpbia, Triceratium y Cerataulus, todos dentro de la familia Biddulphiaceae, establecieron los siguientes grupos taxonómicos de tanto uno como dos géneros:

1) Valvas porosas; marcaciones en las valvas variando gradualmente hasta aquéllas en los procesos o ángulos.

2) Valvas alveoladas; marcaciones en la valva variando gradualmente hacia aquéllas en los procesos o ángulos.

3) Valvas porosas; procesos o ángulos con una placa finamente perforada rodeada por un borde sólido.

4) Valvas alveoladas; procesos o ángulos con una placa finamente perforada rodeada por un borde sólido.

Los primeros dos grupos poseen procesos que son descritos por Anonymous (1975) como pseudocellus, mientras que los procesos del tercer y cuarto grupos se describen como ocellus. Un ocellus (figuras $2,3,4,5,6,7,10$ ) se presenta al final de un cuerno o elevación y es una "placa de sílice, con un borde sin estructura, normalmente grueso, perforada por orificios estrechamente consolidados" (Anonymous, 1975). Un pseudocellus tiene la apariencia superficial de un ocellus, pero es el resultado de la reducción en el tamaño de las areolas a lo largo del cuerno o elevación, lejos de la parte principal de la valva. Esta reducción en el tamaño de las areolas se indica en la elevación izquierda del dibujo de $B$. biddulpbiana (figura 1) y puede verse en los ángulos de la valva de $B$. alternans ilustrada en la figura 15. Ross y Sims (1971) concluyen que el primer grupo (areolas poroides, con pseudocellus) corresponde al género único Biddulpbia; el segundo grupo (areolas alveoladas, con pseudocellus) está limitado al género Trigonium; el tercer grupo (areolas poroides, con ocellus) corresponde al género único Odontella; el cuarto grupo (areolas alveoladas, con ocellus) a los dos géneros Zigoceros y Cerataulus, Sugirieron además, que la clasificación dentro de grupos mayores (familias) se base en la comparación de la estructura de ocellus contra la de pseudocellus, separando así sus géneros de Odontella (junto con Zygoceros y Cerataulus) dentro de la familia Eupodiscaceae y Biddulpbia (junto con Trigonium) dentro de la familia Biddulphiaceae. Las especies originales de Triceratium examinadas, fueron entonces incluidas dentro de todos estos géneros. 
A pesar de que Simonsen (1974) estuvo de acuerdo con Ross y Sims (1971) en que el uso de la simetría de la valva como un carácter taxonómico de diagnóstico había sido "sobre-evaluado", eligió en mantener la distinción entre géneros tri- o poligonales. Aceptó sin embargo, la conclusión de Ross y Sims (1971) de que aquellos géneros que fueron en un principio considerados como pertenecientes a la familia Biddulphiaceae pero que poseen ocelli, deberían de colocarse en la familia Eupodiscaceae. Por lo que muchas de las especies "biddulphioides" que se encuentran dentro de nuestro material de muestreo del Golfo de California, y que fueron tradicionalmente colocadas dentro del género Biddulphia por Hendey (1964) y por Hustedt (1930) deberían de transferirse al género Odontella porque poseen ocelli. Estas especies están registradas en la Tabla 1 junto con sus sinónimos, referencias para identificación y localización dentro de las figuras de este estudio si se ilustran aquí. En esta definición de límites genéricos estamos por lo tanto siguiendo los elementos esenciales de Ross y Sims (1971), observaciones a las que se adhirió Simonsen (1974) para estas especies, y confirmadas por Hoban (1979).

Sabemos que todos los epítetos de especies registradas en la Tabla 1 bajo los géneros Odontella aparecen en la literatura previa excepto $O$. dubia. Hemos decidido transferirla de Biddulpbia dubia después de la comparación de rasgos de diagnóstico en Triceratium dubium (el nombre más antiguo de esta especie), Triceratium favus (el tipo del género) y Biddulpbia dubia, como se ilustra por Allen y Cupp (1935) y que se asemeja muy fuertemente a las características de nuestros especímenes.

Hoban (1979) observó en T. dubium una estructura poroide en la valva y pseudoloculus similares en estructura a aquellos de $T$. favus, pero menos regulares y que se extendían completamente sobre la valva. Un pseudoloculus (figura 2) se describe por Anonymous (1975) como una "cámara formada en el exterior de la capa basal silicea, por expansión de las partes distantes de las costae reticulares o de ramificaciones extremas". Hoban (1979) también observó que las elevaciones de $T$. dubium poseen ocellus. Cupp (1943) trazó su B. dubia con disposición reticular irregular de tamaño desigual en la superficie de la valva, valvas bipolares y con ocellus, con "los lados extendidos para dar un corte transversal cuadrado", y la parte central de la valva convexa. Hendey (1964) afirma que la superficie de la valva de $T$. favus está cubierta con loculus hexagonales regulares, o disposición reticular arreglada en líneas rectas paralelas a los márgenes de la valva. Ross y Sims (1971) reportan también cámaras hexagonales arregladas regularmente en el exterior de la valva de $T$. favus.

Los especímenes de nuestra $O$. dubia del Golfo de California muestran las siguientes características principales. 1) A reolas poroides, 2) disposición reticular ondeada e irregular (pseudoloculae) de tamaño desigual en la superficie de la valva, 3) ocellus presentes en elevaciones opuestas, 4) la valva puede ser de contorno poligonal debido a la extensión de los dos ángulos opuestos que presentan eleva- 
ciones sin ocellus, cada una de las cuales soporta una proyección parecida a un cuerno (figuras 2, 3, 9 y 11). Todas estas características se encuentran en $B$. dubia de Allen y Cupp (1935), y 1), 2), y 3) se comparten con $T$. dubium de Hoban (1979). Sin embargo, nuestros especímenes no poseen loculus de forma hexagonal regular de $T$. favus (Hendey, 1964) y poseen las proyecciones en forma de cuerno de las dos elevaciones que no presentan ocellus; estas proyecciones no se mencionan por Hoban en su descripción de $T$. dubium, ni parecen estar presentes en sus figuras. A pesar de que no hemos examinados nuestros especímenes de Odontella dubia bajo el Microscopio de Barrido Electrónico, creemos que estas proyecciones prominentes son procesos labiales. Deberá también hacerse notar que la descripción de B. dubia dada por Cupp (1943) no está de acuerdo con la de Allen y Cupp (1935). Parece que Cupp malinterpretó la posición de las dos proyecciones con forma de cuerno en su figura 114 , donde aparecen como espinas gruesas, proyectadas cada una desde la base de una elevación con ocellus. Las observaciones anteriores indican que nuestros especímenes son definitivamente la descrita por Allen y Cupp (1935) como B. dubia. Sin embargo, creemos que el hecho de que posea elevaciones con ocellus es requisito para que se le transfiera del género Biddulpbia y se coloque dentro de Odontella.

Dentro del material de muestreo del Golfo de California se han encontrado dos especies con pseudocellus, por lo que deberán colocarse dentro del género Biddulpbia. Estas son $B$. biddulphiana y $B$. alternans que se registran en la Tabla 2 con sus sinónimos más referencias para su identificación y se ilustran en las figuras 1,14 y 15. Nótese que no estamos de acuerdo con Simonsen (1974) en mantener los géneros basándose en la simetría de la valva. B. alternans es tripolar (figura 15) y es equivalen te a Trigonium alternans de Simonsen (1974) y a Triceratium alternans de Hustedt (1930). Posee sin embargo, pseudocellus y características paralelas a las del género Biddulpbia descrito por Hendey (1964). Tiene costae bien desarrolladas y areolas gruesas; aún más, su arreglo de areolas puede ser similar al de $B$. biddulpbiana (que es la especie tipo del género Biddulpbia). Hendey (1964) describe las características de $B$. alternans como "con la valva dividida en tres o más secciones por fuertes costae" que son "generalmente transversales"; y la superficie de la valva entre las costae" con areolas gruesas y redondeadas, reticulares, arregladas en líneas longitudinales o transversales hacia los extremos, pero concéntricas o irregulares en el centro". A Biddulpbia biddulphiana se le da el epíteto de B. pulchella por Hustedt (1930), Simonsen (1974) y por Hendey (1964). Sin embargo, Hoban (1979) deduce que B. biddulpbiana es el nombre correcto y hemos elegido seguir su criterio para el nombre de la especie.

Además de la utilización de los ocelli y los pseudocelli para la separación de los géneros Biddulphia y Odontella (y por ello de sus familias), la estructura de Ios procesos labiales puede ser también útil, aunque Hoban (1979) tiene el cuidado de hacer notar que son menos confiables a nivel de familias. Hoban (comunicación personal) ha notado que los procesos labiales de "la mayoría de las Biddul- 
pbia" no poseen tubos externos, mientras que "la mayoría de las Odontella" si los poseen. Hoban (1979) observó que B. biddulphiana generalmente no posee procesos labiales, pero algunas veces se encuentran dos o seis en la región central de la valva; $B$. alternans posee tres a la mitad de la distancia de una elevación a otra. En Odontella lor procesos labiales son las aparentes espinas largas (figuras 4, 12 y 13; tubos externos) que pueden estar cerca del centro, o cerca del margen de la valva; en las que tanto su número como su colocación puede ser bastante variable dentro de una misma especie.

\section{ACKNOWLEDGEMENTS}

We would like to thank Drs. M. Hoban and G. Fryxell of Texas A \& M University for reviewing an early draft of this work. Their identification of many shortcomings has lead to a great improvement in the final manuscript. We are also greatly indebted to Ms. Clara Yáñez for the exceptional drawings of diatoms illustrated in Figures 1-5. Her observations and detailed sketches helped clarify important details in valve morphology. Mrs. Freda M. H. Reid provided important bibliography and suggestions. Ocean. Vicente Ferreira provided assistence with the photomicrography and Ms. Leticia Amador typed several drafts of the manuscript. This investigation is part of a project supported by CONACYT-BID contract PCMABNA-005321. Financial support was also received from the scholarship program of CONACYT.

\section{AGRADECIMIENTOS.}

Deseamos agradecer a los Dres. M. Hoban y G. Fryxell de la Universidad de Texas A \& M, la revisión de uno de los primeros borradores de este trabajo. Su identificación de muchas omisiones, nos han guiado hacia un gran adelanto en el manuscrito final. Debemos además un gran reconocimiento a la Sra. Clara Yáñez por sus dibujos excepcionales de las diatomeas ilustradas en las Figuras 1-5. Sus observaciones y trazos detallados ayudaron a clarificar detalles importantes en la morfología de la valva. La Sra. Freda M. H. Reid nos proporcionó importante bibliografía y sugerencias. El Ocean. Vicente Ferreira ayudó en la fotomicrografía y la Srita. Leticia Amador mecanografió varios borradores del manuscrito. Esta investigación es parte de un proyecto sostenido por CONACYT-BID contrato PCMABNA-005321. Se recibió además apoyo financiero del programa de becas de CONACYT.

\section{LITERATURE CITED}

ALLEN, W. E. y E. E. Cupp (1935): Plankton diatoms of the Java Sea. Ann. Jard. Bot. Buitenzorg 44, p. 101-174.

ANONYMOUS, 1975. Proposal for a standardization of diatom terminology. Simonsen, R. (Ed.), Beihefte zur Nova Hedwigia, Heft, 53, p. 323-354. 
BRULAND, K. W., 1974. Pb-210 geochronology in the coastal marine environment. Ph. D. Dissertation, University of California, San Diego, 106 p.

CALVERT, S. E., 1964. Factors Affecting Distribution of Laminated Diatomaceus Sediments in Gulf of California. In: Marine Geology of the Gulf of California, T. H. van Andel and G. G. Shor, Jr. (Eds.), American Association of Petroleum Geologists, Memoir 3, p. 311-330.

CALVERT, S. E., 1966. Origin of diatom-rich varved sediments from the Gulf of California. Jour. of Geology, Vol. 76, p. 546-565.

CUPP, E. E., 1943. Marine Plankton Diatoms of the West Coast of North America. SIO Bulletin, vol. 5, No. 1, UC Press, Berkeley, Ca., 238 p.

HANNA, G. D., 1949. A synthetic resin which has unusual properties. J. Roy. Microscopical Soc., LXIX, 25-28.

HENDEY, N. I., 1 964. Bacillariophyceae (Diatoms), In: An Introductory Account of the Smaller Algae of British Coastal Waters. Great Britain Ministry of $\Lambda$ griculture, Fisheries and Food, Series 4, Part 5, 298 p.

HOBAN, M. A., 1979. Morphology, Life Histories and Systematics of Selected Genera of Biddulphioid Diatoms, Ph. D. Dissertation, University of Texas, Austin, $185 \mathrm{p}$.

HOBAN, M. A., G. A., Fryxell, y K. R. Buck, 1980. Bidulphioid Diatoms, Resting Spores in Antartic Eucampia and Odontella. J. Phycol., V. 16, p. 591-602.

HUSTEDT, F., 1927-1930. Die Kieselagen Deutschalands, Osterreichs und der Schweiz mit Beruchsichtigung der ubrigen Lander Europas sowie der angrenzenden Meeresgebiete. In Rabenhorst, L. Kryptogamenflora. Part I, Leipzig.

ROSS, R., y P. A. Sims, 1971. Generic Limits in the Biddulphiaceae as indicated by the Scanning Electron Microscope. In Heywood V. H. (Ed.), Scanning Electron Microscopy, Academic Press, N. Y., p. 155-176.

SIMONSEN, R., 1974. The Diatom Plankton of the Indian Ocean Expedition of R/V "Meteor" 1964-1965. "Meteor" Forsch. Ergebnisse Reihe D. No. 19, p. 1-107. 
TABLE 1

Genus Odontella (Family Eupodiscaceae)

Odontella aurita (Lyngbye) Agardh (Figs. 5, 6, 7, 8)

Agardh 1832, p. 56

Syn: Diatom auritum Lyngbye 1819, p. 182, P1. 62, Fig. D.

Biddulpbia aurita (Lyngbye) de Brebisson 1838, p. 12.

Hustedt, 1930, p. 846, Fig. 501 (as Biddulphia).

Cupp, 1943, p. 161, Figs. 112-A (2), 112-A (3), 112 (B) (as Biddulphia)

Hendey, 1964, p. 103, Pl. 24, Fig. 6 (as Biddulphia).

Hoban, 1979, p. 78, Figs. 58-69 (as Odontella).

Odontella dubia. Comb. nov.

(Figs. 2, 3, 9, 10, 11)

Syn: Biddulpbia dubia (Brightwell) Clcve

Brightwell, 1859, p. 180, Pl. 9, Fig. 12

(Triceratium dubium)

Allen and Cupp, 1935, p. 148, Fig. 84 (as Biddulphia)

Cupp, 1943, p. 164, Fig. 114 (as Biddulpbia)

Hustedt, 1930, p. 278, Figs. 462-463 (as Triceratium dubium)

Hoban, 1979, p. 138, Figs. 163-169 (as Triceratium dubium) 
Odontella mobiliensis (Bailey) Grunow

(Figs. 4, 12, 13)

Grunow 1884, p. 58

Syn: Biddulpbia mobiliensis Grunow in Van Heurck 1882, Pl. 101, Fig. 4-6.

Hustedt, 1930, p. 840, Fig. 495 (as Biddulpbia)

Cupp, 1943, p. 153, Fig. 110 (as Biddulpbia)

Hendey, 1964, p. 104, Pl. 20, Fig. 3 (as Biddulpbia)

Hoban, 1979, p. 118, Figs. 124-136 (as Odontella)

Odontella longicruris (Greville) Hoban

Hoban, 1979, p. 87

Syn: Biddulphia longicruris Greville 1859, p. 163, Pl. 8, Fig. 10

Cupp, 1943, p. 154-156, Fig. 111-A(1-3) (as Biddulphia)

Hoban, 1979, p. 87, Fig. 70-88 (as Odontella)

Odontella regia (Schultze) Simonsen

Simonsen 1974, p. 27

Syn: Biddulpbia regia Ostenfeld, 1908, p. 7, Text. Fig. 3

Hustedt, 1930, p. 383, Fig. 494 (as Biddulpbia)

Hendey, 1964, p. 104, Pl. 20, Fig. 2 (as Biddulpbia)

Hoban, 1979, p. 114, Figs. 117-123 (as Odontella) 
TABLE 2

Genus Biddulpbia

(Family Biddulphiaceae)

Biddulpbia biddulpbiana (J. E. Smith) Boyer (Figs. 1, 14) Boyer, 1900, p. 694.

Syn: Conferva biddulpbiana J. E. Smith 1807, Pl. 1762, upper Fig. Biddulpbia pulcbella Gray 1821, p. 294

Hustedt, 1930, p. 832, Fig. 490 (as Biddulpbia pulcbella)

Cupp, 1943, p. 152, Fig. 109 (as Biddulpbia pulcbella)

Hendey, 1964, p. 101, Pl. 25, Fig. 1 (as Biddulpbia pulcbella)

Hoban, 1979, p. 46, Figs. 4-30 (as Biddulpbia biddulpbiana)

Biddulphia alternans (J. W. Bailey) Van Heurck (Fig. 15)

Van Heurck 1880-1885, p. 208, Pl. 113, Figs. 4, 5, 7.

Syn: Triceratium alternans J. W. Bailey 1851 , p. 14

Trigonium alternans (Bailey) Mann, 1907, p. 290

Hustedt, 1930, p. 825, Fig. 488 (as Triceratium)

Cupp, 1943, p. 155, Fig. 115 (as Biddulpbia)

Hendey, 1964, p. 102, Pl. 25, Fig. 5 (as Biddulpbia)

Hoban, 1979, p. 62, Figs. 31-39 (as Biddulphia) 

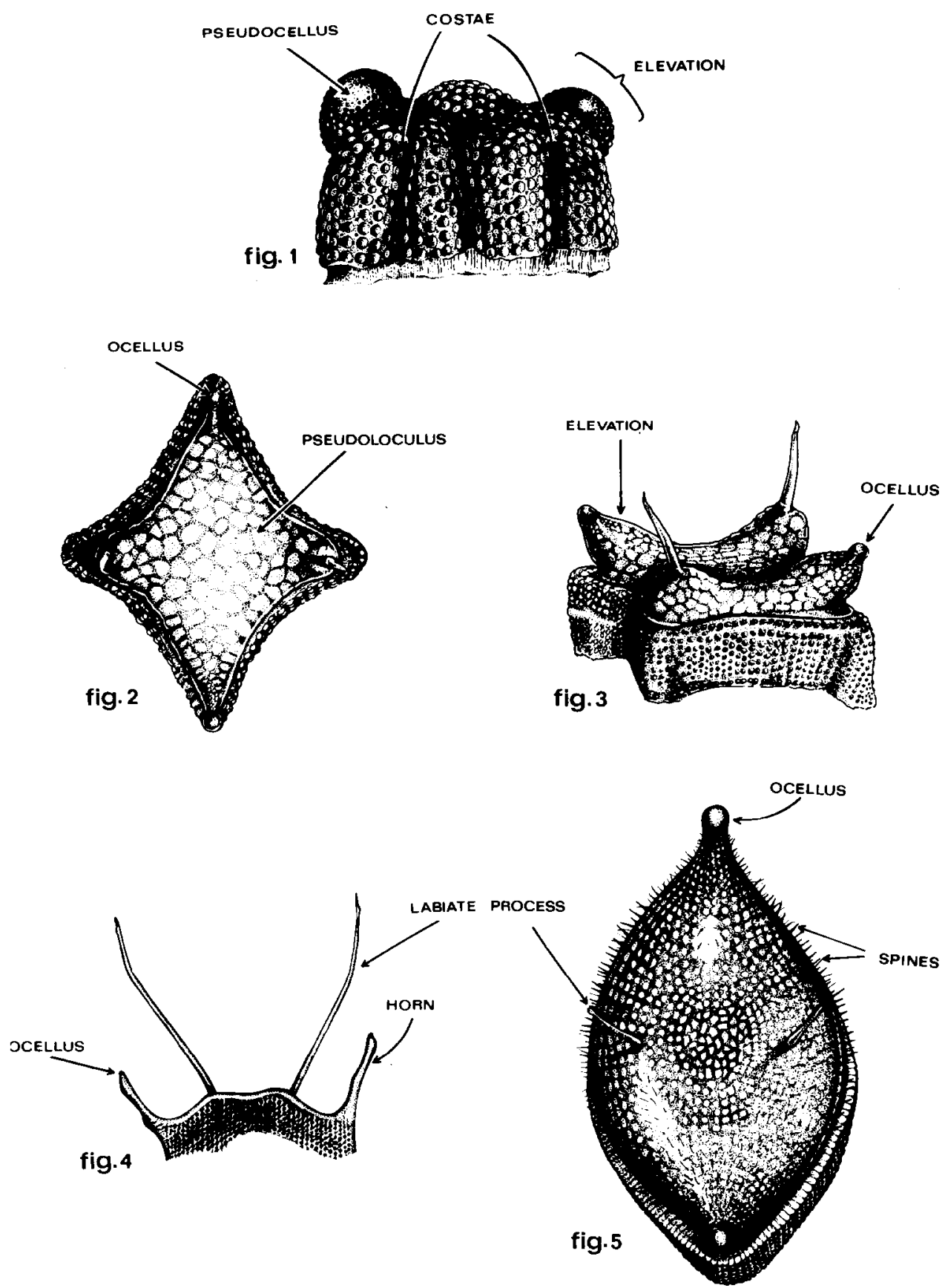


\section{FIGURES 1-5.}

FIGURE 1. Biddulphia biddulphiana. Drawing of a single valve in girdle view, to illustrate the two opposing elevations; the reduction in size of areolae to form the pseudocelli on the elevation; and the costae. This specimen is the same as shown in Figure 14, and has an apical length of $60 \mu m$

FIGURE 2. Odontella dubia. Drawing of the valve surface indicating one of the two opposing ocelli and the pseudoloculli. This is the same specimen as the photomicrograph of figure 9 , and has an apical length of $48 \mu \mathrm{m}$.

FIGURE3. Odontella dubia. Drawing of an inclined valve indicating one of the four elevations and one of the two ocelli. Note the two horn-like projections from the elevations with no ocelli. These projections are thought to be labiate processes.

FIGURE 4. Odontella mobiliensis. Drawing of a single valve in girdle view indicating one of the two horns; one of the two long external labiate processes; and the position of the ocellus on the horn. This is the same specimen as illustrated in figure 12 and has an apical length of $60 \mu \mathrm{m}$.

FIGURE 5. Odontella aurita. Drawing of an inclined valve indicating the position of one of the two ocelli; the numerous spines over the valve surface; and the two long labiate processes. This is the same specimen as that of figure 8 and has an apical length of $58 \mu_{m}$.

\section{FIGURAS 1-5.}

FIGURA 1. Biddulpbia biddulpbiana. Dibujo de una sola valva en vista de cinturón, para ilustrar las dos elevaciones opuestas; la reducción en tamaño de las areolas para formar los pseudocelli en las elevaciones; y la costae. Este espécimen es el mismo que se muestra en la figura 14 , y tiene una longitud de $60 \mu_{m}$ de un ápice al otro.

FIGURA 2. Odontella dubia. Dibujo de la superficie de la valva indicando uno de los dos ocelli opuestos y los pseudoloculli. Este es el mismo espécimen de la fotomicrografía de la figura 9 , y tiene una longitud de $48 \mu^{\mu} m$ de un ápice al otro.

FIGURA 3. Odontella dubia. Dibujo de una valva inclinada, indicando una de las cuatro elevaciones y uno de los dos ocelli. Nótese las dos proyecciones semejantes a cuemos en las elevaciones que no presentan ocelli. Creemos que estas proyecciones son procesos labiales.

FIGURA 4. Odontella mobiliensis. Dibujo de una sola valva en vista de cinturón indicando uno de los dos cuernos; uno de los dos largos externos procesos labiales; y la posición del ocellus en el cuerno. Este es el mismo espécimen que se ilustra en la figura 12 y tiene una longitud de $60 \mu_{m}$ de un ápice a otro.

FIGURA 5. Odontella aurita. Dibujo de una valva indicando la posición de uno de los dos ocelli; las numerosas espinas sobre la superficie de la valva; y los dos largos procesos labiales. Este es el mismo espécimen que el de la figura 8 y tiene una longitud de $58 \mu_{m}$ de un ápice al otro. 
Chávez-Baumgartner

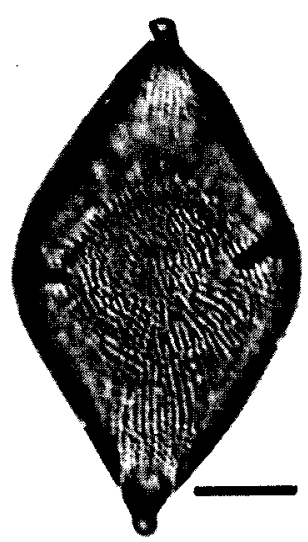

fig. 6

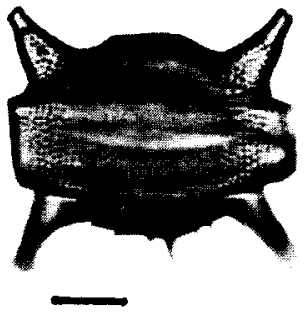

fig. 7

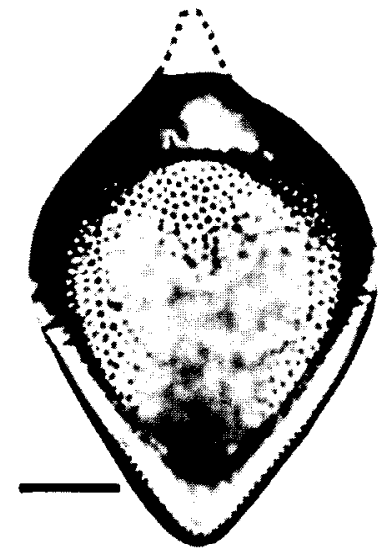

fig. 8

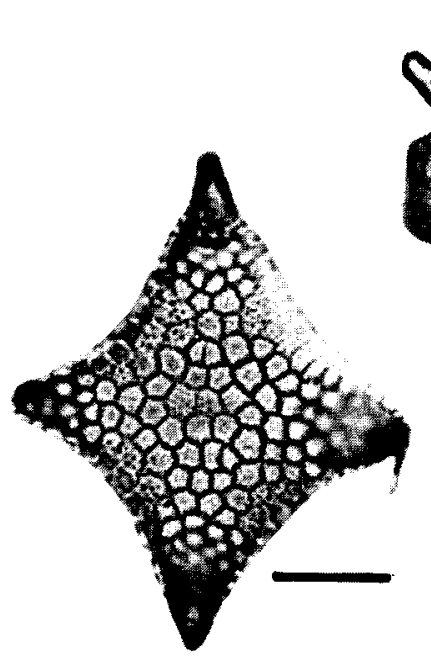

fig. 9

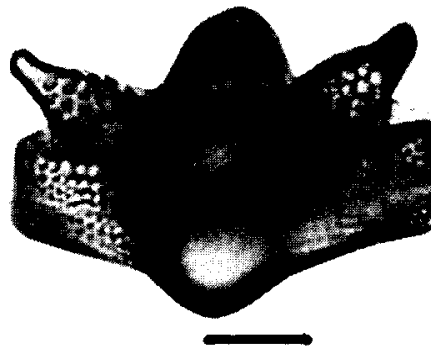

fig. 10

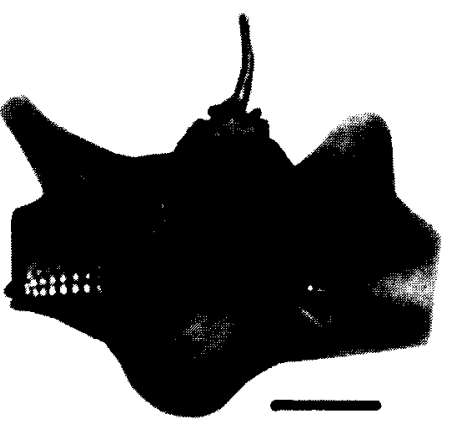

fig. 11 


\section{FIGURES 6-11.}

FIGURE 6. Odontella aurita. Valve view with focus on areolae near convex center of valve. Note ocelli and labiate processes. Scale bar is $10 \mu_{m}$.

FIGURE 7. Odontella aurita. Girdle view of whole frustule with focus on areolae of right elevation on the upper valve. Labiate processes apparently broken off on upper valve. Spines and short process visible on lower valve. Scale bar is $10 \mu \mathrm{m}$.

FIGURE 8. Odontella aurita. Valve surface on inclined specimen. Focus on spines near margin and across central convexity of vaive surface (same specimen as figure 5). Labiate processes and ocelli are not clear from this focus. Scale bar is $10 \mu_{m}$.

FIGURE 9. Odontella dubia. Valve view (compare with figure 2). Focus near center of valve on the poroid areolae. Note the pseudoloculi formed by irregular pattern of ridges across the valve surface. Scale bar is $10 \mu \mathrm{m}$.

FIGURE 10. Odontella dubia. Slightly inclined girdle view of single valve. The two ocellate elevations are shown in this focus. Note pseudoloculli on elevations. Focus is on areolae of valve margin. Scale bar is $10 \mu \mathrm{m}$.

FIGURE 11. Odontella dubia. Same specimen as figure 10 with focus on projection (labiate process?) from non-ocellate elevation and on areolae of valve margin. Scale bar is $10 \mu_{m}$.

\section{FIGURAS 6-11.}

FIGURA 6. Odontella aurita. Vista de valva con foco en las areolas cercanas al centro convexo de la valva. Nótese los ocelli y los procesos labiales. La barra indica una escala de $10 \mu_{m}$.

FIGURA 7. Odontella aurita. Vista de cinturón de una frústula completa con foco en las areolas de la elevación derecha en la valva superior. Los procesos labiales están aparentemente desprendidos de la valva superior. Las espinas y los procesos cortos son visibles en la valva inferior. La barra indica una escala de $10 \mu_{m}$.

FIGURA 8. Odontella aurita. Superficie de la valva de un espécimen inclinado. Con foco en las espinas cercanas al margen y a través de la convexidad central de la superficie de la valva (es el mismo espécimen de la figura 5). Los procesos labiales y los ocelli no son claros desde este foco. La barra indica una escala de $10 \mu_{\mathrm{m}}$. FIGURA 9. Odontella dubia. Vista de valva (compárese con la figura 2). Con foco cerca del centro de la valva en las areolas poroides. Nótese los pseudoloculli formados por un patrón irregular de protuberancias alargadas a través de la superficie de la valva. La barra indica una escala de $10 \mu \mathrm{m}$.

FIGURA 10. Odontella dubia. Vista de cinturón de una sola valva ligeramente inclinada. En este foco se muestran las dos elevaciones con ocellus. Nótese los pseudoloculli en las elevaciones. Con foco en las areolas del margen de la valva. La barra indica una escala de $10 \mu \mathrm{m}$.

FIGURA 11. Odontella dubia. El mismo espécimen que el de la figura 10 con foco en una de las proyecciones ('iproceso labial?) de las elevaciones que no poseen ocellus, y en las areolas del margen de la valva. La barra indica una escala de $10 \mu_{m}$. 
Chávez-Baumgartner

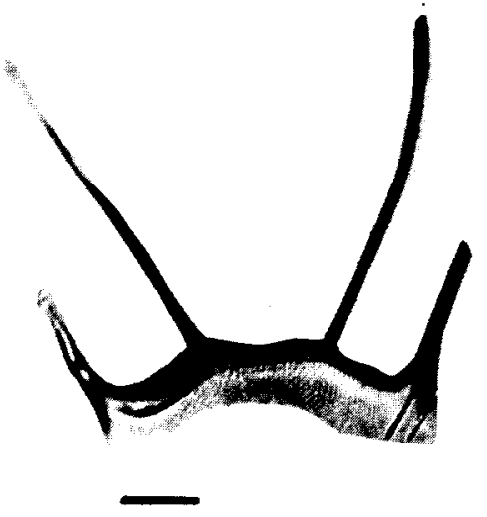

fig. 12

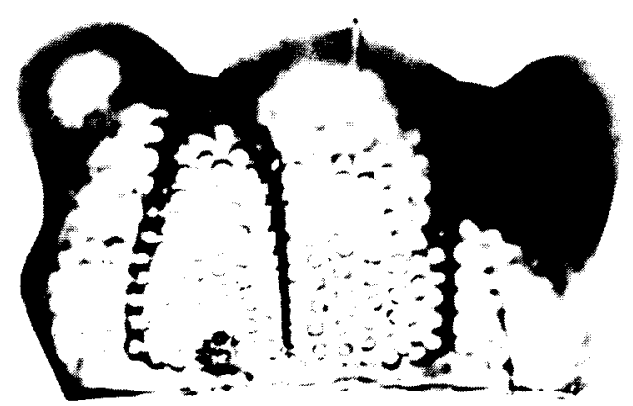

fig. 14

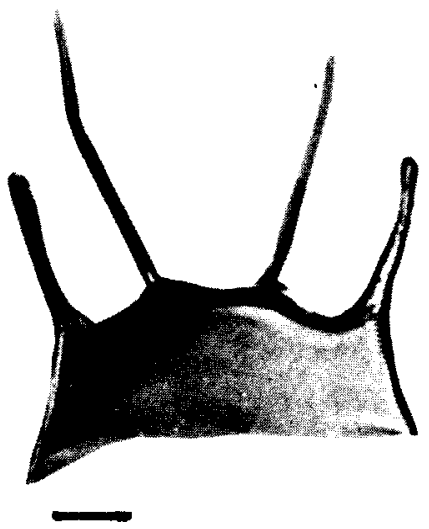

fig. 13

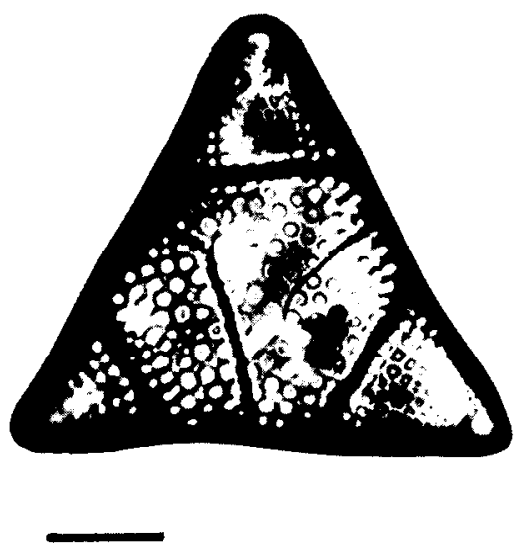

fig. 15 


\section{FIGURES 12-15.}

FIGURE 12. Odontella mobiliensis. Girdle view of single valve (same specimen as drawing of figure 4). Focus on base of rearward labiate process and areolae of valve surface. Scale bar is $10 \mu \mathrm{m}$.

FIGURE 13. Odontella mobiliensis. Girdle view of single valve of different specimen. Focus on forward long labiate process. Scale bar is $10 \mu m$.

FIGURE 14. Biddulpbia biddulpbiana. Girdle view of single valve. Focus on areolae of valve surface. Note costae (compare with figure 1) and the outline (out of focus) of the bulbous elevations. Scale bar is $10 \mu \mathrm{m}$.

FIGURE 15. Biddulpbia alternans. Valve view with tocus on upper and right angles (slight elevations) and areolae in between. Note the reduction of areolae size towards the vertices of the valve angles to form pseudocelli. Scale bar is $10 \mu_{m}$.

\section{FIGURA 12-15.}

FIGURA 12. Odontella mobiliensis. Vista de cinturón de una sola valva (el mismo espécimen que el dibujo de la figura 4). Con foco en la base del proceso labial posterior y en las areolas de la superficie de la valva. La barra indica una escala de $10 \mu_{m}$

FIGURA 13. Odontella mobiliensis. Vista de cinturón de una sola valva de un espécimen diferente. Con foco en el largo proceso labial anterior. La barra indica una escala de 10 um.

FIGURA 14. Biddulpbia biddulphiana. Vista de cinturón de una sola valva. Con foco en las areolas de la superficie de la valva. Nótese las costae (compárese con la figura 1) y el contorno (fuera de foco) de las elevaciones en forma de bulbo. La barra indica una escala de $10 \mu m$.

FIGURA 15. Biddulphia alternans. Vista de valva con foco en los ángulos superior y derecho (ligeras elevaciones) y areolas de enmedio. Nótese la reducción de tamaño de las areolas hacia los vértices de los ángulos de las valvas para formar los pseudocelli. La barra indica una escala de $10 \mu_{m}$ 